\title{
Different Responses of Myocardial and Cerebral Blood Flow to Cord Occlusion in Exteriorized Fetal Sheep
}

\author{
DAVID LEY, GYLFI OSKARSSON, MIKAEL BELLANDER, EDGAR HERNANDEZ-ANDRADE, \\ GÖRAN LINGMAN, KAREL MARSAL, TOMAS OLSSON, ERKKI PESONEN, \\ KRISTINA THORNGREN-JERNECK, OLOF WERNER, AND LENA HELLSTRÖM-WESTAS \\ Department of Pediatrics [D.L., G.O., M.B., E.P., K.T.-J., L.H.-W.], Department of Obstetrics and \\ Gynecology [E.H.-A., G.L., K.M.], Department of Anesthesia and Intensive Care [O.W.], and Department \\ of Radiation Physics [T.O.], Lunds University Hospital, 22185 Lund, Sweden
}

\begin{abstract}
Type and duration of fetal asphyxial insult affect the distribution of blood flow to the heart and brain. The purpose of this study was to describe dynamic and quantitative changes in regional myocardial and cerebral blood flow (CBF) during fetal asphyxia induced by total occlusion of the umbilical cord. Eleven exteriorized fetal sheep were subjected to total umbilical cord occlusion and five fetal sheep served as sham controls. Regional blood flow (BF) to the brain and heart was quantified using radioactive microspheres before and after 5 min of occlusion and finally when fetal mean arterial blood pressure had decreased below $25 \mathrm{~mm} \mathrm{Hg}, 9.8$ (0.8) [mean (SD)] min after occlusion. Right coronary arterial (RCA) blood flow velocity and carotid $\mathrm{BF}$ were registered continuously. Mean values of arterial $\mathrm{pH}$ and oxygen content $\left(\mathrm{mL} \mathrm{O}_{2} / 100 \mathrm{~mL}\right)$ were $7.08(0.11)$ and 4.4 (2.9) before cord occlusion and decreased to $6.83(0.05)$ and $1.4(0.9)$ at 5 min after occlusion ( $p<0.01$, respectively). Carotid BF was significantly below preocclusion values by $2.5 \mathrm{~min}(p<0.05)$, whereas RCA velocity time integral per minute remained above preocclusion values for $9 \mathrm{~min}$. CBF decreased from 316 (24)
\end{abstract}

\section{ABSTRACT}

before cord occlusion to $156(30) \mathrm{mL} / \mathrm{min} / 100 \mathrm{~g}$ at $5 \mathrm{~min}(p<$ 0.01 ), whereas right myocardial BF was maintained at 792 (125) and 751 (183) $\mathrm{mL} / \mathrm{min} / 100 \mathrm{~g}$, respectively. CBF decreased rapidly after total cord occlusion whereas myocardial BF increased and was maintained until shortly before cardiac arrest, suggesting the myocardium to be better preserved during this type of insult in already partially asphyxiated fetuses. (Pediatr Res 55: 568$575,2004)$

\section{Abbreviations}

MABP, mean arterial blood pressure

CBF, cerebral blood flow

BF, blood flow

Art HT, arterial hypotension

$\mathrm{PaCO}_{2}$, arterial $\mathrm{CO}_{2}$ pressure

EEG, electroencephalography

RCA, right coronary artery

PFV, peak flow velocity

VTI, velocity time integral
The vascular response of the heart and brain to acute asphyxia in the late gestation fetus varies according to type and rapidity of insult. As opposed to the centralization of flow in fetal hypoxemia with a well-established compensatory increase to the brain and heart, acute asphyxia may result in a variety of vascular responses. Total cord occlusion has resulted in an early decrease in carotid blood flow, suggesting a vulnerability of the fetal brain during an early stage of the insult (1). The

Received August 1, 2002; accepted September 18, 2003.

Correspondence: David Ley, M.D., Ph.D., Department of Pediatrics, Lunds University Hospital, 22185 Lund, Sweden; e-mail: david.ley@skane.se

Supported by the Swedish Medical Research Council, the Swedish Heart Lung Foundation, the Royal Swedish Physiographic Society, the Crafoord Foundation, the Swedish Heart-Children's Association, the University Hospital of Lund, and the Medical Faculty of Lund University.

DOI: 10.1203/01.PDR.0000113785.66455.E7 decrease in carotid blood flow and cerebral blood volume was accompanied by an increase in carotid vascular resistance.

Localization of regional brain damage has been attributed to differences in timing of the asphyxial insult $(2,3)$. The regional vascular response within the brain during the asphyxial insult has been sparsely investigated. Thus, differences in vascular reactivity within the brain due to variations in type and timing of the insult may predispose to selective regional vulnerability of brain structures.

A previous study reporting an early decrease in carotid blood flow during acute asphyxia with maintained blood pressure suggested that this decrease was not due to cardiac compromise (1). We postulated that myocardial and cerebral perfusion are differentially regulated during acute asphyxia. Studies of early concomitant changes in $\mathrm{CBF}$ and myocardial $\mathrm{BF}$ in response to sustained periods of total cord occlusion have not been per- 
formed. Such study requires a combination of methods to enable both detection of rapid dynamic changes as well as quantification of absolute values of regional blood flow. A continuous assessment of relative changes in CBF may be performed by measuring carotid $\mathrm{BF}$, whereas a similar evaluation of myocardial perfusion requires continuous information on coronary blood flow. This may be achieved by measurements of RCA Doppler velocity. The method has been applied in the human fetus showing signs of increased coronary perfusion in chronic uteroplacental insufficiency (4). Fetal coronary flow dynamics have not previously been studied during acute asphyxia.

The objective of this study was to investigate the dynamic changes of the fetal cerebral and coronary circulations as well as absolute changes in blood flow within the brain and heart in response to acute asphyxia, as induced by umbilical cord occlusion. For this purpose we used intermittent measurements of cerebral and myocardial BF by radiolabeled microspheres, and continuous measurements of carotid BF and RCA Doppler flow velocity.

\section{METHODS}

Subjects and surgery. The study was performed in 16 near-term fetal sheep of mixed breed and gender at a mean (range) gestational age of $136(134-138)$ d. Gestational age was calculated from the day of conception. The study was approved by the Animal Ethics Research Committee, Lunds University.

The pregnant ewes were intubated after pentobarbitone induction and cesarean section was performed during isoflurane anesthesia supplemented by remifentanil infusion. Fetuses were exteriorized into a thermostated waterbath at $39^{\circ} \mathrm{C}$. Care was taken to minimize disturbance of the umbilical circulation and to maintain body temperature and humidity. To avoid fetal breathing, the nose was covered with a rubber glove. Catheters were placed in the femoral and axillary arteries with the tips located in the abdominal aorta and the brachiocephalic trunk, respectively. Another catheter was placed in the femoral vein with the tip located in the inferior vena cava. An ultrasound blood flow probe (3S, Transonic Systems Inc., Ithaca, NY, U.S.A.) was placed around the carotid artery for measurement of carotid blood flow. Two EEG electrodes (shielded stainless steel) were placed bilaterally over the parietal cortex $10 \mathrm{~mm}$ anterior of the bregma and $15 \mathrm{~mm}$ lateral of the midline after making drill holes through the parietal bone. A reference electrode was placed subcutaneously, posteriorly in the midline of the skull.

Recordings and measurements. MABP in the aorta via the femoral catheter, heart rate derived from pressure oscillations, and carotid BF were registered continuously. Heart rate and MABP signals were stored on hard disk. Single-channel EEG was recorded and stored on hard disk with continuous display of the raw EEG and the amplitude-integrated EEG. Arterial acid-base measurements were performed in blood obtained from the abdominal aorta via the femoral catheter and analyzed immediately on the ABL 300 (Radiometer, Copenhagen, Denmark). Blood oxygen saturation and $\mathrm{Hb}$ concentration were measured by an OSM 2 (Radiometer) hemoximeter and used to calculate oxygen content. Blood gases were corrected for fetal temperature. Arterial acid-base measurements were performed before cord occlusion, at $5 \mathrm{~min}$ after occlusion, and when fetal MABP had decreased below $25 \mathrm{~mm} \mathrm{Hg}$.

Doppler ultrasound blood flow velocities in the RCA were registered with a high-frequency linear transducer (15L8), Acuson Sequoia C256 (Siemens, Mountain View, CA, U.S.A.). The ultrasound transducer was placed on the right side of the thoracic wall of the lamb fetus, which allowed visualization of the proximal right coronary artery at its take off from the ascending aorta (Fig. 1). After identifying the RCA by two-dimensional echocardiography and color flow mapping, flow velocity was registered continuously in the proximal part of the right coronary artery with $7.0 \mathrm{MHz}$ pulsed wave Doppler ultrasound. The angle between the Doppler beam and the longitudinal axis of the vessel was kept as small as possible, and was always $<15^{\circ}$. Angle correction was not performed. A sample volume that gave the best possible quality envelope and pure audio sound throughout the cardiac cycle was chosen. All examinations were recorded on super-VHS videotape and selected still frames on magneto-optic disc. Measurements of Doppler velocities and time integrals were performed off-line, with the analysis package of the ultrasound unit. Coronary flow is dependent upon the pressure gradient across the coronary bed and therefore occurs mainly during diastole, with systolic flow being considerably less and even negligible (5). Coronary flow is best estimated by adjusting the diastolic VTI for heart rate, thereby correcting for changes in VTI caused by lengthening or shortening of the cardiac cycle ( $\mathrm{R}-\mathrm{R}$ interval). Diastolic PFV and VTI were measured on three to five cardiac cycles and the results averaged. VTI per minute was calculated by multiplying VTI with heart rate. Rate pressure product was calculated by multiplying systolic blood pressure with heart rate.

Regional blood flow to the brain and heart was measured by using the reference organ radiolabeled microsphere technique (6). Microspheres, $15 \mu \mathrm{m}$ in diameter (New England Nuclear, Zaventem, Belgium) and labeled with one of three isotopes (cerium 141, scandium 46, and chromium 51) were injected into the fetal inferior vena cava over a 20 -s period while reference samples where obtained from the brachiocephalic trunk and the abdominal aorta at a rate of $3.3 \mathrm{~mL} / \mathrm{min}$ for 1.5 min using a precalibrated pump (Harvard Apparatus, Dover, MA, U.S.A.). The volume of blood removed for flow reference samples was replaced with an equal volume of maternal blood. After completion of the study, the fetal brain and heart were removed and fixed in formaldehyde. The brain was dissected into the following regions: frontal, parietal, temporal, and occipital cortex; hippocampus; diencephalon and striatum; pons, cerebellum, and medulla oblongata. The heart was dissected in myocardial sections from the right and left chamber. The radioactivity in each sample was determined using a Ge-detector (Canberra Industries Inc., Meriden, CT, U.S.A.) with the Genie 2000 (Canberra Packard, Schwadorf, Austria ) analysis software. Activity in all calculated samples represented an excess of 400 microspheres. The mean of calculated blood flows in all regions, excluding those to brain stem, pons 

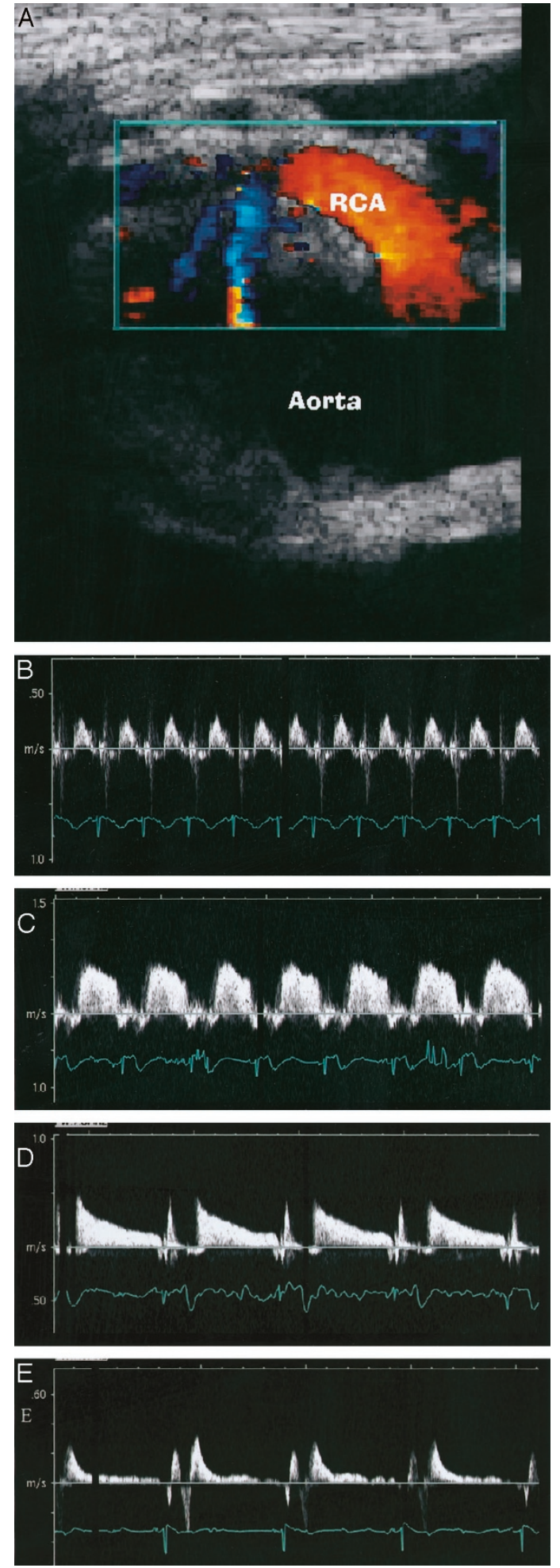

Figure 1. $(A)$ Illustration of the proximal RCA visualized by color Doppler at its take off from the ascending aorta. $(B-E)$ Illustration of characteristic sequence of changes in the RCA Doppler velocity spectrum before and during total cord occlusion; $(B)$ before cord occlusion; $(C)$ increased RCA Doppler velocity at 3 min after cord occlusion; $(D)$ and $(E)$ decreasing RCA Doppler velocities at 7 and $10 \mathrm{~min}$, respectively, after cord occlusion.

and cerebellum, were taken to represent cerebral blood flow. Regional BF was calculated for the following regions: basal ganglia (mean of diencephalon and striatum), cortex (mean of frontal, temporal, parietal, and occipital cortex), hippocampus and infratentorial brain structures (mean of medulla oblongata, pons, and cerebellum).

Experimental procedure. After exteriorization and surgical preparation, fetuses were allowed $20 \mathrm{~min}$ of stabilization, after which baseline measurements were performed. Thereafter, total umbilical cord occlusion was performed in 11 lambs by an inflatable cuff. Physiologic data were monitored until cardiac arrest as defined by ultrasound imaging (i.e. absence of ventricular contractions). Doppler blood flow velocities in the RCA were registered directly after exteriorization of the fetus, intermittently during preparation and stabilization, and continuously during umbilical cord occlusion. Microsphere measurements were performed before cord occlusion, after $5 \mathrm{~min}$ of occlusion, and finally when fetal MABP had decreased below $25 \mathrm{~mm} \mathrm{Hg}$. MABP below $25 \mathrm{~mm} \mathrm{Hg}$ was taken as a definition of Art HT. After an identical exteriorization and surgical preparation, five lambs served as sham controls and were monitored continuously during $10 \mathrm{~min}$. Microsphere measurements in sham controls were performed at 0,5 , and $10 \mathrm{~min}$.

Statistics. Results are presented as mean \pm SD unless otherwise specified. Data were analyzed using one-way repeated-measures ANOVA. Correlations between parameters were assessed using Pearson's correlation analysis. $p$ Values $<0.05$ were considered significant.

\section{RESULTS}

Fetal sheep subjected to cord occlusion were acidotic and moderately hypoxic before the onset of cord occlusion as were sham controls throughout the protocol. During cord occlusion, the fetuses developed severe combined metabolic and respiratory acidosis. Physiologic data of fetal sheep subjected to cord occlusion and those of sham controls are given in Table 1.

Fetal heart rate fell rapidly after the start of cord occlusion, whereas mean MABP initially increased and was maintained for $4.5 \mathrm{~min}$ compared with preocclusion values and then decreased continuously until cardiac arrest (Fig. 2). Art HT (i.e. MABP $<25 \mathrm{~mm} \mathrm{Hg}$ ) was determined at a mean (SD) of 9.8 (0.8) min and cardiac arrest occurred at 14.5 (4.2) min after onset of occlusion. MABP and acid-base variables remained unchanged in the sham controls during the study period. The EEG became isoelectric at a mean time of 68 (19) sec after onset of occlusion and remained so until cardiac arrest.

Cerebral blood flow. Carotid BF exhibited an early decrease and fell significantly below preocclusion values by $2.5 \mathrm{~min}(p$ $<0.01$ ) (Fig. 2). Mean values of regional CBF in asphyxiated lambs before and during cord occlusion as well as in sham controls are given in Table 2. Mean (SD) relative decrease in blood flow compared with preocclusion values at 5 min was $40 \%(17 \%)$ in cortical areas, $24 \%(23 \%)$ in the hippocampus, $44 \%(14 \%)$ in basal ganglia, and 48\% (12\%) in infratentorial brain structures and 79\% (15\%), 73\% (20\%), 79\% (14\%), and $82 \%(10 \%)$ respectively at Art HT. There were no significant differences in relative changes in blood flow between the respective cerebral brain regions at either 5 min or at Art HT compared with preocclusion values. 
Table 1. Physiological variables in fetal sheep subjected to cord occlusion and in sham controls

\begin{tabular}{|c|c|c|c|c|c|c|}
\hline & \multicolumn{3}{|c|}{ Asphyxiated fetal sheep $(n=11)$} & \multicolumn{3}{|c|}{ Sham controls $(n=5)$} \\
\hline & Baseline & $5 \min$ & Art HT & Baseline & $5 \mathrm{~min}$ & $10 \mathrm{~min}$ \\
\hline $\mathrm{pH}$ & $7.08 \pm 0.11$ & $6.83 \pm 0.05 \dagger$ & $6.80 \pm 0.06 \S$ & $7.08 \pm 0.14$ & $7.07 \pm 0.14$ & $7.07 \pm 0.15$ \\
\hline Base excess $(\mathrm{mmol} / \mathrm{L})$ & $-9.8 \pm 5.1$ & $-18.1 \pm 3.0 \dagger$ & $-18.8 \pm 2.4 \S$ & $-10.3 \pm 7.6$ & $-10.9 \pm 6.9$ & $-10.7 \pm 7.2$ \\
\hline Oxygen content $\left(\mathrm{mL} \mathrm{O}_{2} / 100 \mathrm{~mL}\right)$ & $4.4 \pm 2.9$ & $1.4 \pm 0.9 \dagger$ & $0.8 \pm 0.3 \S$ & $4.0 \pm 2.0$ & $4.1 \pm 1.8$ & $4.0 \pm 2.3$ \\
\hline MABP $(\mathrm{mm} \mathrm{Hg})$ & $45 \pm 3$ & $28 \pm 3 \dagger$ & $13 \pm 1 \|$ & $53 \pm 5$ & $53 \pm 4$ & $50 \pm 4$ \\
\hline
\end{tabular}

Values are mean $\pm \mathrm{SD}$. Art HT was defined when MABP had decreased below $25 \mathrm{~mm} \mathrm{Hg}$ during cord occlusion.

$* p<0.05$ and $\dagger p<0.01$, significant decrease at 5 min as compared with baseline; $\$ p<0.05$ and $\S p<0.01$, significant decrease at Art HT as compared with baseline; $\| p<0.01$, significant decrease at Art HT as compared with at $5 \mathrm{~min}$ after cord occlusion.

Myocardial blood flow. Mean values of right and left myocardial $\mathrm{BF}$ as measured by microspheres in asphyxiated lambs before and during cord occlusion as well as in sham controls are given in Table 2. Blood flow to the right myocardium was significantly higher than that to the left myocardium before cord occlusion and remained so at $5 \mathrm{~min}$ and at Art HT ( $p<$ 0.05. respectively). Mean values of $\mathrm{BF}$ to the right and left myocardium remained unchanged at $5 \mathrm{~min}$ after cord occlusion and decreased significantly at Art HT compared with the preocclusion values $(p<0.01)$.

RCA Doppler flow velocity dynamics. RCA hemodynamic parameters for asphyxiated fetal sheep and sham controls are summarized in Table 3. The lowest PFV, VTI, and VTI per minute were obtained directly after exteriorization. During the period from exteriorization to umbilical cord occlusion there was a significant increase in PFV, VTI, and VTI per minute ( $p$ $<0.05$, respectively). Correlation analyses performed on data obtained before umbilical cord occlusion showed a negative correlation between PFV and $\mathrm{pH}(r=-0.68, p=0.0003)$, and a weaker $(r=-0.47$ to -0.59$)$ but significant $(p<0.05)$ negative correlation between PFV and both oxygen content and base excess. There were no significant correlations between RCA flow parameters and heart rate, blood pressure, or the rate pressure product.

After umbilical cord occlusion, there was an immediate increase in PFV, VTI, and VTI per minute (Fig. 1). PFV and VTI per minute fell below preocclusion values after 5 and 9 min, respectively. Compared with data obtained immediately after exteriorization, the maximal PFV after umbilical cord occlusion was 3.04 (1.79) times higher and the maximal VTI per minute 4.17 (2.22) higher. Changes in VTI per minute in the asphyxiated group before and during cord occlusion are shown in Figure 2.

Right myocardial blood flow versus RCA Doppler flow velocity parameters. Correlation analyses showed a linear correlation between right ventricular $\mathrm{BF}$ as measured by microspheres and RCA VTI per minute $(r=0.69, p<0.0001)$ as registered by Doppler ultrasound (Fig. 3). There was a similar linear correlation between right ventricular BF and RCA PFV $(r=0.63, p<0.0001)$.

Myocardial versus cerebral blood flow. The mean (SD) ratio of volume blood flow as measured by microspheres, between that of the right myocardium and that of the cerebrum [right myocardium $(\mathrm{mL} / \mathrm{min} / 100 \mathrm{~g}) /$ cerebrum $(\mathrm{mL} / \mathrm{min} / 100 \mathrm{~g})$ ] increased significantly from that before cord occlusion, 2.4
(1.0), to that at 5 min after cord occlusion, $4.3(1.7)(p<0.01)$, whereas the ratio at Art HT, 3.7 (2.1), did not differ significantly from the preocclusion ratio. The corresponding values in sham controls were $2.4(0.8)$ at $0 \mathrm{~min}, 2.8(0.8)$ at $5 \mathrm{~min}$, and $3.1(0.3)$ at $10 \mathrm{~min}$. Relative changes in carotid BF, RCA flow velocity (VTI per minute), and MABP during cord occlusion are given in Figure 4, illustrating the respective variables temporal relationship to changes in MABP with an early decrease in carotid $\mathrm{BF}$ compared with an increase and preservation of RCA flow velocity.

\section{DISCUSSION}

The main purpose of this study was to compare changes in time and magnitude of differences in cerebral and myocardial $\mathrm{BF}$ during acute asphyxia induced by total cord occlusion. The results show that the vascular responses in the heart and brain to asphyxia of rapid onset are fundamentally different. We observed an immediate fall in carotid BF after onset of occlusion associated with an abrupt increase in carotid vascular resistance. On the contrary, RCA velocity increased abruptly after onset of occlusion, indicating a clear fall in coronary vascular resistance, and reached values below those before occlusion at 5 min after onset of occlusion. The CBF measured by the microsphere technique showed that $\mathrm{CBF}$ was reduced by approximately $50 \%$ after 5 min of occlusion compared with preocclusion values, whereas right and left myocardial blood flows were unchanged. When fetal MABP had decreased below $25 \mathrm{~mm} \mathrm{Hg}$ (i.e. at the point of Art HT), CBF was reduced by $80 \%$ and myocardial BF by $75 \%$ compared with preocclusion values. Although fetal hypoxemia induces an increase in $\mathrm{CBF}$ compared with the decrease observed in asphyxia of rapid onset, the differential response between the myocardial and cerebral vasculature is similar in that both insults lead to a greater increase in myocardial $\mathrm{BF}$ than in $\mathrm{CBF}$ (7).

Methodological considerations. The microspheres technique is limited in that it only allows for intermittent measurements of $\mathrm{BF}$, and it is difficult to obtain samples quickly enough to determine rapid changes. This is well illustrated by our observation that the microspheres method failed to detect the rapid increase in RCA flow as detected by the continuous Doppler registration after onset of cord occlusion. The combined use of continuous coronary Doppler and carotid flow measurements with intermittent measurements of blood flow using the microspheres method enabled an evaluation of dy- 

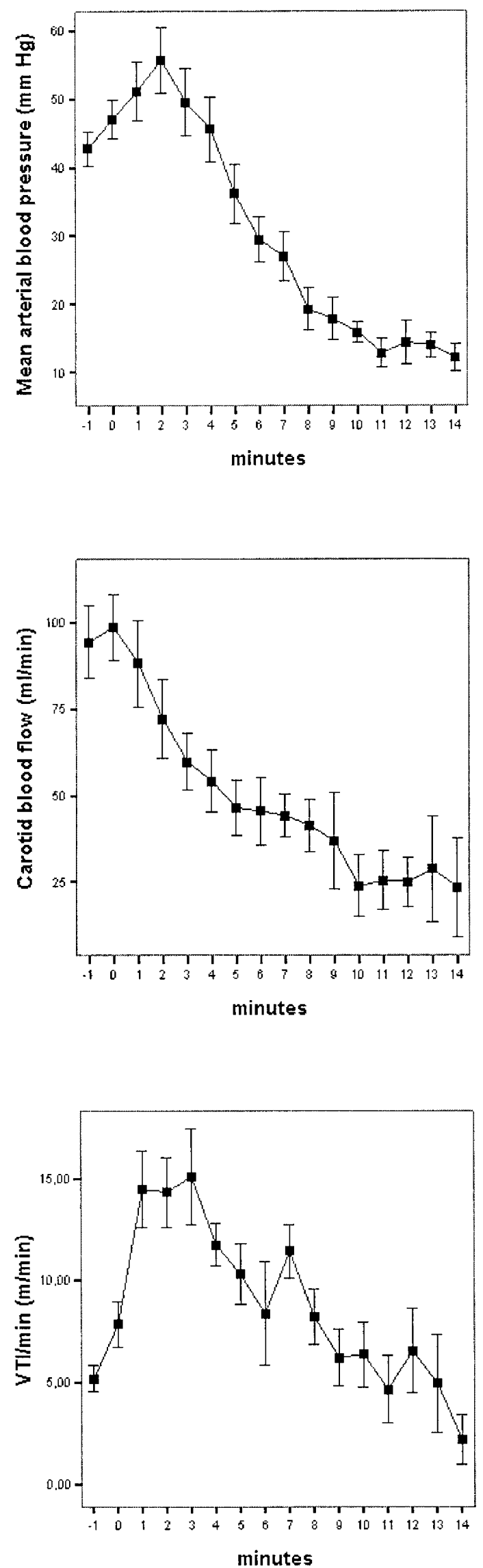

Figure 2. Mean values of MABP (top), CBF (middle), and VTI per minute (bottom) in fetal sheep $(n=11)$ subjected to total cord occlusion. Error bars denote SEM. namic changes substantiated by absolute measures at defined time points.

A fairly good linear relationship was found in our study between right coronary Doppler parameters and right myocardial $\mathrm{BF}$, and a good correlation has previously been found between carotid $\mathrm{BF}$ and $\mathrm{CBF}$ as measured by microspheres (8). The Transonic flow probe was used as a continuous measure of changes in cerebral blood flow. Branches from the carotid artery perfusing extracerebral tissue were not ligated, therefore changes in perfusion to extracerebral tissue such as a rapid fall to carcass tissue may have masked an early increase in cerebral blood flow (9). A reciprocal distribution of flow between cerebral and extracerebral structures with increasing carbon dioxide tensions has been noted in the fetal sheep without any measurable change in blood flow as measured with a flow probe on the common carotid artery (10). Bennet et al. (1) reported a similar early fall in carotid blood flow, as in the present study, during cord occlusion in fetal sheep with ligated extracerebral branches of the carotid artery as well as in those without ligated branches, suggesting that the proportion of volume flow to extracerebral tissue and the presumed decrease thereof was of a less important magnitude.

A prerequisite for transthoracic measurements of RCA velocity of good quality was exteriorization of the fetus. This procedure was associated with the development of a degree of fetal acidemia and hypoxemia before onset of cord occlusion. The baseline values of BF to supra- and infratentorial brain regions as well as those to the heart in the present study are 1.5to 2-fold higher than corresponding baseline values for unanesthetized, chronically instrumented sheep fetuses (11-13). The increased values of $\mathrm{BF}$ to the heart and brain before cord occlusion represent a fetal response to metabolic acidosis and hypoxemia and might in turn have affected the subsequent hemodynamic response to total cord occlusion. The characteristic differences observed between myocardial and cerebral BF during cord occlusion must therefore be interpreted as a vascular response in which both organs have been subjected to hypoxia and acidosis before the acute asphyxial insult.

Cerebrovascular response. Our findings are similar to those of a recent study in near-term fetal sheep showing a similar rapid fall in carotid $\mathrm{BF}$ and an increase in carotid vascular resistance in response to total cord occlusion (1). The response to cord occlusion of rapid onset is distinctly different to that of hypoxia, where a number of studies have shown a response characterized by a compensatory increase in CBF and a preservation of oxygen delivery to the brain $(14,15)$. The fall in carotid BF was in all lambs preceded by the EEG becoming isoelectric, which implies that the latter is an early response to a decrease in oxygenation and not to a decrease in CBF. Arterial blood pressure fell below baseline value at a mean time of 4 min after onset of cord occlusion, which is significantly earlier than that $(8 \mathrm{~min})$ reported in the study of Bennet et al. (1). The shorter duration of the hypertensive phase in the present study was probably due to fetal hypoxemia and acidemia before cord occlusion, which in turn may have induced loss of cerebral autoregulatory capacity (11). This being the case, autoregulation of the cerebral vasculature would appear 
Table 2. Regional blood flow to brain and heart as measured by radioactive microspheres in fetal sheep subjected to cord occlusion and in sham controls

\begin{tabular}{|c|c|c|c|c|c|c|}
\hline \multirow[b]{2}{*}{ Regional blood flow (mL/min/100 g) } & \multicolumn{3}{|c|}{ Asphyxiated fetal sheep $(n=11)$} & \multicolumn{3}{|c|}{ Sham controls $(n=5)$} \\
\hline & Baseline & $5 \min$ & Art HT & Baseline & $5 \mathrm{~min}$ & $10 \mathrm{~min}$ \\
\hline Cerebrum & $316 \pm 24$ & $156 \pm 30 \dagger$ & $58 \pm 16 \S \|$ & $277 \pm 33$ & $283 \pm 53$ & $263 \pm 47$ \\
\hline Cortex & $249 \pm 23$ & $118 \pm 23 \dagger$ & $41 \pm 12 \S \|$ & $202 \pm 23$ & $211 \pm 37$ & $200 \pm 32$ \\
\hline Hippocampus & $312 \pm 31$ & $178 \pm 40 \dagger$ & $67 \pm 18 \S \|$ & $287 \pm 36$ & $276 \pm 54$ & $260 \pm 56$ \\
\hline Basal ganglia & $453 \pm 29$ & $221 \pm 41 \dagger$ & $86 \pm 24 \S \|$ & $423 \pm 65$ & $430 \pm 88$ & $389 \pm 75$ \\
\hline Infratentorial brain & $596 \pm 39$ & $282 \pm 56 \dagger$ & $106 \pm 29 \S \|$ & $633 \pm 52$ & $646 \pm 117$ & $548 \pm 68$ \\
\hline Right heart ventricle & $792 \pm 125$ & $751 \pm 183$ & $214 \pm 59 \S \|$ & $668 \pm 135$ & $791 \pm 157$ & $830 \pm 171$ \\
\hline Left heart ventricle & $598 \pm 115$ & $529 \pm 132$ & $150 \pm 48 \S \|$ & $483 \pm 94$ & $626 \pm 132$ & $678 \pm 164$ \\
\hline
\end{tabular}

Values are mean $\pm \mathrm{SD}$. Art HT was defined when MABP had decreased below $25 \mathrm{~mm} \mathrm{Hg}$ during cord occlusion.

$* p<0.05$ and $\dagger p<0.01$, significant decrease at 5 min as compared with baseline; $\$ p<0.05$ and $\S p<0.01$, significant decrease at Art HT as compared with baseline; $\| p<.01$, significant decrease at Art HT as compared with at 5 min after cord occlusion.

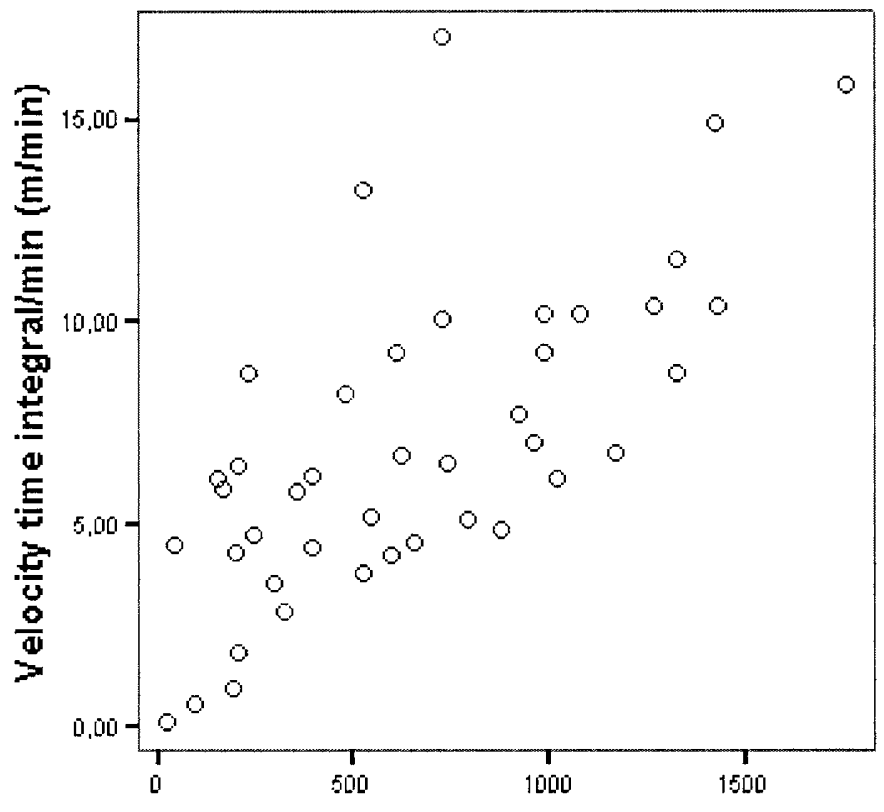

Right myocardial blood flow (ml/min/100g)

Figure 3. Correlation between right myocardial $\mathrm{BF}$ as measured by radioactive microspheres and RCA VTI per minute as measured by Doppler ultrasound.

more vulnerable than that of the heart in face of the differentiated hemodynamic responses we observed.

The reason for the sharp increase in cerebrovascular resistance during cord occlusion of rapid onset is unclear. A predominant feature of cord occlusion is an early increase in fetal $\mathrm{PaCO}_{2}$ due to immediate cessation of placental gas exchange. Recent studies have shown that superimposed excessive hypercapnia in rats subjected to cerebral ischemia and hypoxia induces severe cerebrovascular depression and aggravates brain damage (16). The influence of $\mathrm{PaCO}_{2}$ on cerebrovascular response and cerebral damage in sheep occlusion models is not known and warrants further study. If elevated $\mathrm{PaCO}_{2}$ would be found to induce an increase in cerebrovascular resistance during cord occlusion, this would be contrary to previous findings of cerebral vasodilatory effects of hypercapnia in the sheep fetus (17).

A previous study using microspheres showed no change in CBF during total arrest of uterine blood flow, with a redistri-

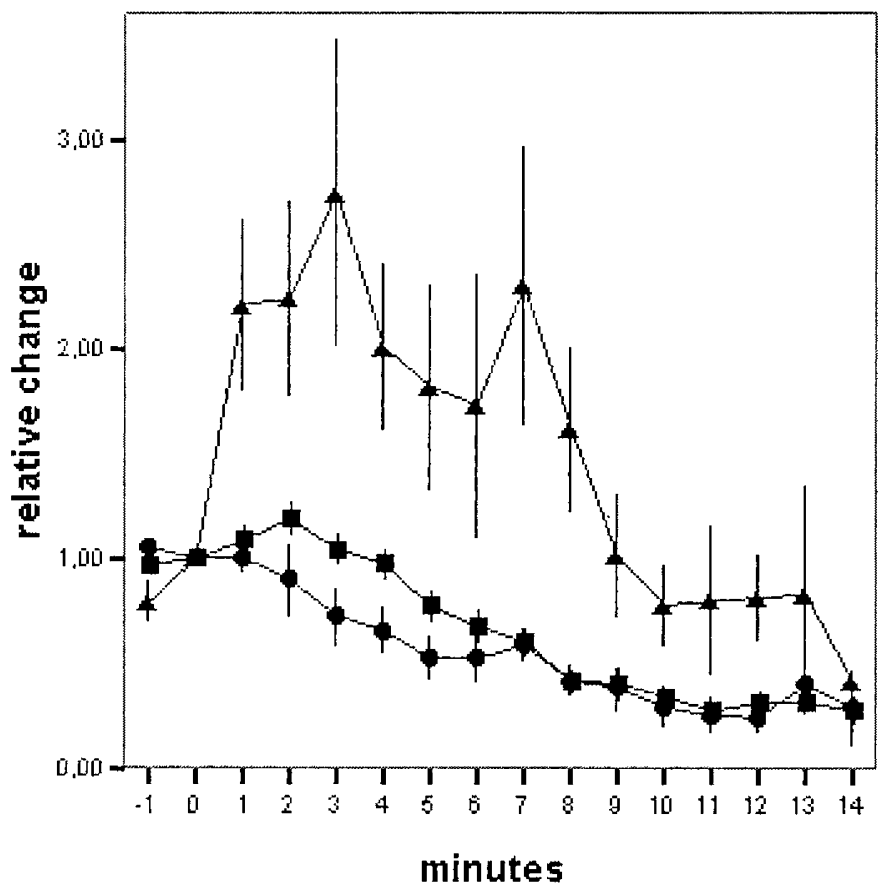

Figure 4. Mean relative changes in $\operatorname{MABP}(\square), \operatorname{CBF}(\bullet)$, and RCA VTI per minute $(\mathbf{\Delta})$ in fetal sheep $(n=11)$ subjected to total cord occlusion. Error bars denote SEM.

bution within the brain favoring basal structures (13). We were unable to observe any sign of redistribution within the brain as the relative decrease in perfusion to supra- and infratentorial brain structures was similar at $5 \mathrm{~min}$ and at Art HT. The hierarchy of regional perfusion was preserved at the three time points of microsphere administration and corresponds to the degree of metabolism normally attributed to basal, supra-, and infratentorial structures. Information on any concurrent changes in regional oxygen extraction or metabolism was not obtained in this study which further precludes speculation on regional vulnerability.

Coronary blood flow. High coronary flow velocity in human fetuses has been observed in uteroplacental insufficiency, and identifies fetuses at high risk of intrauterine death $(4,18)$. Studies on coronary blood flow in fetal lambs using external Doppler flow probes placed on the left circumflex artery and radiolabeled microspheres have been performed during right 
Table 3. Right coronary Doppler flow parameters in the asphyxiated lambs, registered directly after exteriorisation, before and during umbilical cord occlusion

\begin{tabular}{|c|c|c|c|c|}
\hline & \multicolumn{3}{|c|}{ Asphyxiated lambs } & \multirow{2}{*}{$\frac{\text { Sham controls }}{\text { Baseline to birth }}$} \\
\hline & Initial & Baseline & Peak flow & \\
\hline $\mathrm{PFV}(\mathrm{cm} / \mathrm{s})$ & $30.3 \pm 13.7$ & $47.8 \pm 22.0^{*}$ & $72.2 \pm 30.3 t t$ & $45.7 \pm 17.5$ \\
\hline $\mathrm{VTI} / \mathrm{min}(\mathrm{m} / \mathrm{min})$ & $4.65 \pm 1.82$ & $8.20 \pm 3.87 *$ & $17.21 \pm 6.96 \dagger \S$ & $6.33 \pm 2.43$ \\
\hline Mean BP (mm Hg) & $46 \pm 8$ & $43 \pm 9$ & $47 \pm 15$ & $53 \pm 11$ \\
\hline Heart rate $(\mathrm{b} / \mathrm{min})$ & $187 \pm 37$ & $201 \pm 33$ & $105 \pm 53 \dagger t$ & $196 \pm 16$ \\
\hline
\end{tabular}

The peak flow after umbilical cord occlusion was obtained at mean $3 \mathrm{~min}$ (range 1-10 min). The flow parameters presented for the sham controls are average values of those obtained during exteriorization. Data are presented as mean $\pm \mathrm{SD}$.

${ }^{*} p<0.05$ significant difference at baseline as compared with initial; $\uparrow p<0.05$ significant difference at peak flow compared with baseline; $\ddagger p<0.01$, significant difference at peak flow as compared with initial, $\S p<0.001$, significant difference at peak flow as compared with initial.

ventricular pressure loading and pure hypoxemia $(19,20)$. Acute systolic pressure loading of the right ventricle to its maximal work capability stimulated coronary flow to the double, whereas acute hypoxemia caused a 4-fold increase in flow $(19,20)$.

This study showed that acute asphyxia induced by total cord occlusion caused a rapid increase in RCA flow, and RCA flow was sustained above baseline values for 5-9 $\mathrm{min}$. When RCA flow had decreased to preasphyxia values, the fetal lambs were on the verge of cardiac arrest.

The maximal increase in RCA flow velocity during asphyxia was 4-fold compared with measurements made immediately after exteriorization of the lambs. This increase is comparable to the maximal flow increase caused by pure hypoxemia in previous studies (19). The increase in RCA flow in this study was, however, larger than the flow increase obtained in a previous study by pressure loading of the fetal right ventricle (20).

The relationship between left and right ventricular myocardial $\mathrm{BF}$ at rest was comparable with previous studies in fetal lambs, left ventricular blood flow being two thirds to three quarters of the right ventricular blood flow (21). This relationship remained unchanged during cord occlusion, even at severe Art HT. Thus, the hemodynamic and metabolic changes induced by cord occlusion appear to have a similar effect on left and right ventricular myocardial $\mathrm{BF}$.

The hemodynamic changes caused by umbilical cord occlusion are characterized by immediate bradycardia, initial increase in arterial blood pressure, and successive arterial hypotension. In a previous study, a close relationship was shown between the rate-pressure product (product of heart rate and systolic blood pressure) and right ventricular myocardial BF (20). This relationship could not be found in our flow data obtained before umbilical cord occlusion. After umbilical cord occlusion, the rate-pressure product fell directly whereas RCA flow increased rapidly. Under the influence of acute asphyxia, neither heart rate nor blood pressure correlated with RCA flow, and it is more likely that a metabolic response such as the release of adenosine in the myocardium due to reduced oxygen content caused the rapid increase observed in RCA flow (22).

The metabolic changes caused by umbilical cord occlusion are characterized by metabolic acidosis, hypercapnia, and hypoxemia. Each of these parameters may affect coronary blood flow, and a previous study has shown fetal coronary blood flow velocity to be closely related to oxygen content (19). In this study, data obtained before umbilical cord occlusion showed a rather weak negative correlation between oxygen content and RCA peak flow velocity, whereas a stronger negative correlation was found between RCA peak flow velocity and arterial $\mathrm{pH}$. Acidosis has not previously been shown to affect fetal coronary blood flow, but as hypoxemia and acidosis coincided in this study, a causal relationship between acidosis and increased fetal coronary flow velocity could not be verified.

The compensatory increase in RCA velocity and the preservation of myocardial BF showed that the coronary vessel bed reacted distinctly differently to abrupt asphyxia compared with the cerebral vasculature. The early decrease in CBF associated with an increase in cerebrovascular resistance is characteristic of insults of rapid onset and has not been observed in studies with slower or graded decreases of umbilical or uterine BF (12, 23). Sheep fetuses were acidemic and hypoxic before the acute asphyxial insult and therefore the differentiated vascular response to cord occlusion represents that of the partially asphyxiated fetus and not necessarily that of the healthy fetus. We argue that these observations are of value for physiologic understanding in a clinical setting as it is frequently perceived that human fetuses are in poor metabolic condition before acute asphyxia.

Histologic study of organ damage in near-term fetal lambs subjected to total cord occlusion showed that changes in the myocardium were only seen in those fetuses with the most severe histologic brain damage, suggesting the myocardium to be better preserved than the brain during this type of insult (24). Similarly, studies in newborn infants with severe hypoxic ischemic encephalopathy have shown varied degrees of signs of myocardial ischemia as reflected in blood chemistry (25). The likely explanation for these findings in cases of acute obstruction of blood flow through the umbilical cord is an increased preservation of myocardial BF compared with that of $\mathrm{CBF}$, thus rendering the brain ischemic during a longer time period than the heart.

Acknowledgments. The authors thank Ingela MattissonSandström, Ulla Ganestam, Laura Darcy, Ann-Christine Andersson, Annica Maxedius, and Michaela Merglova for excellent assistance during the experiments. 


\section{REFERENCES}

1. Bennet L, Peebles DM, Edwards DA, Rios A, Hanson MA 1998 The cerebral hemodynamic response to asphyxia and hypoxia in the near-term fetal sheep as measured by near infrared spectroscopy. Pediatr Res 44:951-957

2. Mallard EC, Gunn AJ, Williams CE, Johnston BM, Gluckman PD 1992 Transient umbilical cord occlusion causes hippocampal damage in the fetal sheep. Am J Obste Gynecol 167:1423-1430

3. Mallard EC, Williams CE, Gunn AJ, Gunning MI, Gluckman PD 1993 Frequent episodes of brief ischemia sensitize the fetal sheep brain to neuronal loss and induce striatal injury. Pediatr Res 33:61-65

4. Baschat A, Gembruch U, Reiss I, Gortner L, Diedrich K 1997 Demonstration of fetal coronary blood flow by Doppler ultrasound in relation to arterial and venous flow velocity waveforms and perinatal outcome - the 'heart-sparing effect.' Ultrasound Obstet Gynecol 9:162-172

5. Oskarsson G, Pesonen E 2000 Coronary flow abnormalities in neonates with aortic stenosis. J Pediatr 137:875-877

6. Heymann MA, Payne BD, Hoffmann JIE, Rudolph AM 1977 Blood flow measurements with radionuclide-labeled microspheres. Prog Cardiovasc Dis 10:55-79

7. Rurak DW, Richardson BS, Patrick JE, Carmichael L, Homan J 1995 Blood flow and oxygen delivery to fetal organs and tissues during sustained hypoxemia. Am J Physio $269 \cdot \mathrm{H} 2074-\mathrm{H} 208$

8. van Bel F, Roman C, Klautz RJM, Teitel DF, Rudolph AM 1994 Relationship between brain blood flow and carotid arterial flow in the sheep fetus. Pediatr Res 35:329-333

9. Richardson BS, Carmichael L, Homan J, Johnston L, Gagnon R 1996 Fetal cerebral, circulatory and metabolic responses during heart rate decelerations with umbilical cord compression. Am J Obstet Gynecol 175:929-936

10. Dunnihoo DR, Quilligan EJ 1973 Carotid blood flow distribution in the in utero sheep fetus. Am J Obstet Gynecol 116:648-655

11. Tweed WA, Cote J, Pash M, Lou H 1983 Arterial oxygenation determines autoreg ulation of cerebral blood flow in the fetal lamb. Pediatr Res 17:246-249

12. Ball RH, Parer J, Caldwell LE, Johnson J 1994 Regional blood flow and metabolism in ovine fetuses during severe cord occlusion. Am J Obstet Gynecol 171:1549-1555
13. Jensen A, Hohmann M, Kunzel W 1987 Dynamic changes in organ blood flow and oxygen consumption during acute asphyxia in fetal sheep. J Dev Physiol 9:543-559

14. Cohn HE, Sacks EJ, Heymann MA, Rudolph AM 1974 Cardiovascular responses to hypoxemia and academia in fetal lambs. Am J Obstet Gynecol 120:817-824

15. Peeters LLH, Sheldon RE, Jones JR, Makowski EL, Meschia G 1979 Blood flow to fetal organs as a function of arterial oxygen content. Am J Obstet Gynecol 135:637646

16. Vannuci RL, Towfighi J, Brucklacher RM, Vannucci SJ 2001 Effect of extreme hypercapnia on hypoxic brain damage in the immature rat. Pediatr Res 49:799-803

17. Rosenberg AA, Jones MD Jr, Traystman RJ, Simmons MA, Molteni RA 1982 Response of cerebral blood flow to changes in $\mathrm{PCO}_{2}$ in fetal, newborn and adult sheep. Am J Physiol 242:H862-H866

18. Chaoui R 1997 The fetal 'heart-sparing effect' detected by the assessment of coronary blood flow: a further ominous sign of fetal compromise. Ultrasound Obstet Gynecol 9:5-9

19. Reller M, Burson M, Lohr J, Morton M, Thornburg K 1995 Nitric oxide is an important determinant of coronary flow at rest and during hypoxemic stress in fetal lambs. Am J Physiol 269:H2074-H2081

20. Reller M, Morton M, Giraud G, Wu D, Thornburg K 1992 Severe right ventricular perfusion loading in fetal sheep augments global myocardial blood flow to submaximal levels. Circulation 86:581-588

21. Fisher DJ, Heymann MA, Rudolph AM 1980 Myocardial oxygen and carbohydrate consumption in fetal lambs in utero and in adult sheep. Am J Physiol 238:H399$\mathrm{H} 405$

22. Mainwaring R, Mentzer R, Ely S, Rubio R, Berne R 1985 The role of adenosine in the regulation of coronary blood flow in newborn lambs. Surgery 98:540-546

23. Ball RH, Parer JT, Espinoza M, Alon E, Vertommen J, Johnson J 1994 Regional blood flow in asphyxiated fetuses with seizures. Am J Obstet Gynecol 170:156-16

24. Ikeda T, Murata Y, Quilligan E, Parer J, Murayama T, Koono M 2000 Histological and biochemical study of the brain, heart, kidney and liver in asphyxia caused by occlusion of the umbilical cord in near-term fetal lambs. Am J Obstet Gynecol 182:449-457

25. Barberi I, Calabro M, Cordaro S, Gitto E, Sottile A, Prudent D, Bertuccio G, Consolo S 1999 Myocardial ischemia in neonates with perinatal asphyxia. Electrocardiographic, echocardiographic and enzymatic correlations. Eur J Pediatr 158:742747 